\title{
Quantum Control for Trapped Particles at Matter Surface
}

\author{
Quan-Fang Wang* \\ The Chinese University of Hong Kong \\ E-mail: quanfangwang9@gmail.com
}

In this theoretic work, trapped particles at matter surface to be considered as target system of quantum control. At the framework of variational method in Hilbert space, it would be quite interesting for us to explore particles which is trapped via optical lattice or other kinds of constraints at a matter surface (metal, crystal). The aim of this task is to survey theoretical control for quantum particles as they are appeared and trapped at matter surface (cf. [1]). The physical background of this work is laying on the specified particles motion or reaction under a certain chemical surface. As is well known, one can move a particle at surface smoothly through a point force above it or according to a proper angle, such quantum mechanical motion had already been achieved by the IBM team several years ago.

At the viewpoint of quantum control, what is theoretic support? Can we make these control theoretically, computationally or experimentally? In fact, free trapped particles had been considered by scientists and researchers at worldwide scale. The most exciting things in this study is to take particle as target as it constrained on a surface. Theoretically, this work is to describe quantum control system consisting of time-varying Schrödinger equation at physical constraints condition. Then to apply control theory to quantum system of trapped

\footnotetext{
*To whom correspondence should be addressed
} 
particles, find and characterize optimal quantum control. Further, to compose optimality system (Euler-Lagrange system). Comprising of control free trapped particle, this work is focusing on control taking place at matter surface (on it particle is trapped), that is, try to discuss the external force constrain (e.g. optical lattice) and surface constrain are acting at particle together.

Amazing result is desired in control of different multi-forces as control inputs, what would be happened as a particle changing its position, displacement or status under trapped situation? can we make a trapped chemical quantum well, or a physical optical lattice which worked using external force? what is extension of such kind of works at a variety of fields? whether the general quantum control is worked in this case? It is the purpose to solve these mysteries in this work, and report the initial conclusion of theoretic aspect for trapped particle at matter surface.

\section{Density Function Theory (DFT)}

Particles control could been treated using density function theory (DFT) to measure the motion of electrons surrounding it citing [2],[3] abstracts. Although considered particle at a certain matter surface, in the case of no chemical reaction happening at control time duration, and neglect the motion of vertical direction, then it make target particle laying on a two-dimensions spatial space (as usual, supposed as plane surface). It can be regarded using 2 dimensional polar coordinate.

Let $\Omega$ be an open bounded set of $\mathbf{R}^{2}$ at polar coordinate $\mathbf{r} \in(0, \infty)$, vector $\mathbf{r}$, and $Q=$ $(0, T) \times \Omega$ for $T>0,(t, \mathbf{r}) \in Q$. The ground state energy density of electron is denoted as $\eta(t, \mathbf{r}), \mathbf{r}$ is the displacement of electron from centre of a particle, $\vec{E}(t, \overrightarrow{\mathbf{r}})$ is electronic field. Then time-dependent density functional theory has the form of Schödinger equation (cf. [4])

$$
\mathbf{i} \frac{\partial}{\partial t} \eta(t, \mathbf{r})=\left[-\frac{1}{2} \nabla^{2}+\int \frac{\eta\left(t, \mathbf{r}^{\prime}\right)}{\left|\mathbf{r}-\mathbf{r}^{\prime}\right|} d \mathbf{r}^{\prime}+\frac{\delta E_{x c}(\eta(t, \mathbf{r}))}{\delta(t, \mathbf{r})}\right.
$$




$$
\left.-\vec{E}_{0}(t) \cdot \overrightarrow{\mathbf{r}}-\vec{E}_{s}(t, \overrightarrow{\mathbf{r}}) \cdot \overrightarrow{\mathbf{r}}+\vec{F}(t, \overrightarrow{\mathbf{r}}) \cdot \overrightarrow{\mathbf{r}}\right] \eta(t, \mathbf{r})
$$

In here, $\eta(t, \mathbf{r})$ is wave function of particle, represent the probability function at time $t$. $E_{x c}(\eta(t, \mathbf{r}))$ is exchange correction function. $\vec{E}_{s}(t, \overrightarrow{\mathbf{r}})$ or $\vec{E}_{s}(t)$ is (Raman) scattered field. $\vec{F}(t, \overrightarrow{\mathbf{r}})$ is time-spatial-varying function representing constrained force of the particle, such as optical lattice, trapped laser pulse, etc. The formula of $\vec{F}(t, \overrightarrow{\mathbf{r}})$ is depended on the electromagnetic apparatus at physics laboratory, such as lattice structure (vary optical lattice depth, tune the running lattice frequency), lattice number, dipole, etc. For example, in the case of optical trapped, we need to consider the photon electron (photon energy for clean $\operatorname{GaAs}(110)(1 \times 1)$ surface, $\operatorname{Si}(111)(7 \times 7)$ surface) interaction (cf. [5]) for detailed formulation of the external force $\vec{F}$. Definitely, without lost of generality, assume $F$ is correlated to time $t$ and spatial variable $\mathbf{r}$. Notice that constrained force $\vec{F}$ at cooling technique is positive for electron density represented particle system, it restored the symmetric forces. The Hamiltonian of particle in the presence of incident field $\vec{E}_{0}(t)$ at time-depended density functional theory (TDDFT, cf. [6]) lead the form of

$$
\begin{aligned}
\hat{H}(t)= & -\frac{1}{2} \nabla^{2}+\int \frac{\eta\left(t, \mathbf{r}^{\prime}\right)}{\left|\mathbf{r}-\mathbf{r}^{\prime}\right|} d \mathbf{r}^{\prime}+\frac{\delta E_{x c}(\eta(t, \mathbf{r}))}{\delta(t, \mathbf{r})} \\
& -\vec{E}_{0}(t) \cdot \overrightarrow{\mathbf{r}}-\vec{E}_{s}(t, \overrightarrow{\mathbf{r}}) \cdot \overrightarrow{\mathbf{r}}+\vec{F}(t, \overrightarrow{\mathbf{r}}) \cdot \overrightarrow{\mathbf{r}}
\end{aligned}
$$

Constant $E_{0}(0)$ is energy of the particle (ion, atom) at their equilibrium position.

Remark 1. DFT use the polar coordinate $\overrightarrow{\mathbf{r}}=\left(\vec{r}_{1}, \vec{r}_{2}\right)$ is slightly different (vectors superposition $\overrightarrow{\mathbf{r}}=\vec{r}_{1}+\vec{r}_{2}$ for $r_{1}, r_{2} \in(0, \infty)$, and $\left.\mathbf{r} \in(0, \infty)\right)$ from mathematics polar coordinate $(r, \theta): x=r \sin \theta, y=r \cos \theta$. Cite 3D vector polar coordinate in Figure 1. 


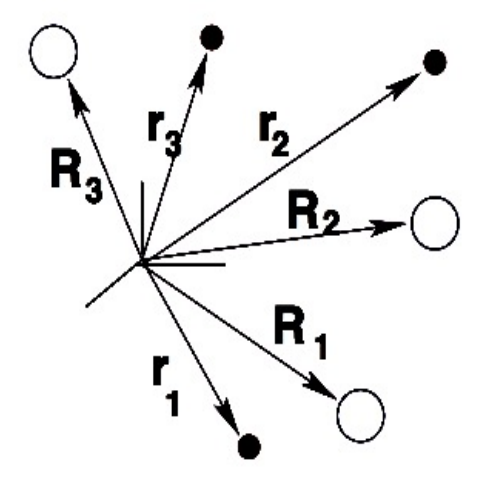

Fig.1 Polar coordinate: $3 \mathrm{D} \overrightarrow{\mathbf{r}}=\left(\vec{r}_{1}, \vec{r}_{2}, \vec{r}_{3}\right), \overrightarrow{\mathbf{R}}=\left(\vec{R}_{1}, \vec{R}_{2}, \vec{R}_{3}\right)$.

Remark 2. Make constrained force to be a constraint condition. At orthogonal coordinate, constrained force which making particle stationary at surface should be satisfied the condition at each coordinate axis is 0 , at the polar coordinate, particle position is the origin, then constrained vector force $\vec{F}(t, \overrightarrow{\mathbf{r}})$ acting at electron (nucleus), and scattered force $E_{s}(t, \overrightarrow{\mathbf{r}})$ (include $\left.E_{0}(t)\right)$ should make total force as null. Abstract formulated from quantum mechanics such as $\vec{E}_{0}(t)+\vec{E}_{s}(t, \overrightarrow{\mathbf{r}})=\vec{F}(t, \overrightarrow{\mathbf{r}}), \frac{\partial \hat{H}}{\partial \mathbf{r}}=0$ or their weak form, there is no experimental setup and data, could not make detail sense at current stage. Hence, our particle force $\vec{F}$ is a kind of universal at the moment of theoretically controlling. Put numerics calculation aside at initial theoretic work, left to be involved in the computational trapping. In fact, set Lagrangian function

$$
\begin{aligned}
\mathcal{L}(\sigma)= & \mathbf{i} \frac{\partial \eta}{\partial t}-\left[-\frac{1}{2} \nabla^{2}+\int \frac{\eta}{\left|\mathbf{r}-\mathbf{r}^{\prime}\right|} d \mathbf{r}^{\prime}+\frac{\delta E_{x c}(\eta)}{\delta(t, \mathbf{r})}-\vec{E}_{0} \cdot \overrightarrow{\mathbf{r}}-\vec{E}_{s} \cdot \overrightarrow{\mathbf{r}}+\vec{F} \cdot \overrightarrow{\mathbf{r}}\right] \eta \\
& +\sigma\left[\vec{E}_{0}+\vec{E}_{s}-\vec{F}\right] .
\end{aligned}
$$

to solve the parameter optimization problems. It is accordance to quantum mechanics theory.

$$
\left\{\begin{array}{l}
\mathbf{i} \frac{\partial \eta(t, \mathbf{r})}{\partial t}=\frac{\partial \hat{H}}{\partial t} \eta(t, \mathbf{r}) \\
\frac{\partial \hat{H}}{\partial \mathbf{r}}=0
\end{array}\right.
$$

To get the minima of Hamiltonian for space variable $\mathbf{r}$, and keep minimizing energy at status 
of stationary of particles at surface.

\section{Time-Depended Schrödinger Equation}

For theoretic work, consider many-body $N$ particles (ion ${ }^{171} \mathrm{Yb}^{+}$, atom ${ }^{87} \mathrm{Rb}$ ) at a (rigid solid) surface (e.g. Iron $\mathrm{Fe}(23)$ at copper $\mathrm{Cu}(111)$ surface; hydrogen $\mathrm{H}$ atom at tungsten W(001) surface). Suppose particle at its equilibrium position (fixed temporally) at optical lattice. A description for chemical calculation could be configured of lattice, reaction (absorption, desorption), and velocity ratio. The initial kinetic energy, trajectory, projection, particle motion can be caught up using optical control. There is insufficient energy in the motion system, particle desorb independently, the trajectory resulted in particle being trapped on the surface at either insufficient energy or insufficient component of momentum to escape from the surface. The rigid surface which is not allow transfer of energy from particle to surface, vice verse, therefore, any particles no absorb no desorb at a trajectory, its means that particle is supposed to be trapped, lasting a observable time duration. Neglecting the velocity of vertical direction component, it should not affect the shape of opacity function at laboratory.

Set $\mathbf{n}(t, \mathbf{r})=\sum_{i=1}^{N}\left|\eta^{i}(t, \mathbf{r})\right|^{2}$ (same calculation for vector $\overrightarrow{\mathbf{n}}(t, \mathbf{r})=\left(\eta^{1}(t, \mathbf{r}), \eta^{2}(t, \mathbf{r}), \cdots\right.$, $\left.\eta^{N}(t, \mathbf{r})\right)$. For simplification, suppose the force $\vec{F}(t, \overrightarrow{\mathbf{r}})$ is equal for $N$ particles at a certain time $t$. Hence, time-depended Schrödinger equation take the sum form of

$$
\begin{aligned}
\mathbf{i} \frac{\partial}{\partial t} \mathbf{n}(t, \mathbf{r})= & -\frac{1}{2} \nabla^{2} \mathbf{n}(t, \mathbf{r})+[\mathbf{e}(t, \mathbf{r})+\mathbf{V}(t, \mathbf{r}) \\
& -\mathbf{g}(t) \mathbf{r}+\mathbf{f}(t, \mathbf{r}) \mathbf{r}] \mathbf{n}(t, \mathbf{r})
\end{aligned}
$$

In here, $\mathbf{e}(t, \mathbf{r})=\int \frac{\mathbf{n}\left(t, \mathbf{r}^{\prime}\right)}{\left|\mathbf{r}-\mathbf{r}^{\prime}\right|} d \mathbf{r}^{\prime}$ is electron-electron interaction, $\mathbf{V}(t, \mathbf{r})=\frac{\delta E_{x c}(\mathbf{n}(t, \mathbf{r}))}{\delta(t, \mathbf{r})}$ is the contribution of exchange correction functional $E_{x c}$. For polar vector $\overrightarrow{\mathbf{r}}=\left(\vec{r}_{1}, \vec{r}_{2}\right)^{t}$, set $\vec{E}_{0}(t)=\left(g_{0}(t), \cdots, g_{0}(t)\right)^{t}$ and unified scattered electric field $\vec{E}_{s}(t)=\left(g_{s}(t), \cdots, g_{s}(t)\right)^{t}$ 
(same probability to scatter to arbitrary direction at arbitrary angle), then denote $\mathbf{g}(t) \mathbf{r}=$ $\vec{E}_{0}(t) \cdots \overrightarrow{\mathbf{r}}+\vec{E}_{s}(t) \cdot \overrightarrow{\mathbf{r}}$ is external electronic field for $N$ particles. Similarly, to a particle, assume $\mathbf{f}(t, \mathbf{r})$ is constrained force (trapped potential $\mathbf{F}=\left(F^{1}, F^{2}, \cdots, F^{N}\right), F^{i}$ is $i$ th ion or atom) having same probability to act at the particle at arbitrary direction and position, i. e. $\vec{F}(t, \overrightarrow{\mathbf{r}})=(f(t, \overrightarrow{\mathbf{r}}), \cdots, f(t, \overrightarrow{\mathbf{r}}))^{t}$, hence denote $\mathbf{f}(t, \mathbf{r}) \mathbf{r n}(t, \mathbf{r})=\sum_{i=1}^{N} \vec{F}(t, \overrightarrow{\mathbf{r}}) \cdot \mathbf{r} \eta^{i}(t, \mathbf{r})$.

For different density functional model, $E_{x c}$ of $\mathbf{V}(t, \mathbf{r})$ has difference at Thomas-Fermi (TF) model (cf. [7],[8]), Thomas-Fermi-Dirac (TFD) model (cf. [9]), and Thomas-Fermi DiracWeizsäcker (TFDW) model (cf. [10]). In here, $(\hbar) h$ is (reduced) Planck constant, $m$ is electron mass.

$$
\begin{aligned}
\mathbf{V}(t, \mathbf{r}) & =\frac{\delta E_{x c}(\mathbf{n}(t, \mathbf{r}))}{\delta(t, \mathbf{r})}=\frac{5}{3} c_{k} \int \mathbf{n}(\mathbf{r})^{2 / 3} d \mathbf{r}+v(\mathbf{r}) \quad \mathrm{TF} \\
& =\frac{5}{3} c_{k} \int \mathbf{n}(\mathbf{r})^{2 / 3} d \mathbf{r}+v(\mathbf{r})-\frac{4}{3} c_{e} \int \mathbf{n}(\mathbf{r})^{\frac{1}{3}} d \mathbf{r} \mathrm{TFD} \\
& =\frac{5}{3} c_{k} \int \mathbf{n}(\mathbf{r})^{2 / 3} d \mathbf{r}+v(\mathbf{r})-\frac{4}{3} c_{e} \int \mathbf{n}(\mathbf{r})^{\frac{1}{3}} d \mathbf{r} \\
& +\frac{1}{8}\left(\frac{\hbar^{2}}{m}\right) \int\left[\frac{\left|N \mathbf{n}(\mathbf{r})^{2}\right|}{\mathbf{n}(\mathbf{r})}-2 \frac{|N \mathbf{n}(\mathbf{r})|}{\mathbf{n}(\mathbf{r})}\right] d \mathbf{r}, \quad \mathrm{TFDW}
\end{aligned}
$$

$c_{k}=\frac{3}{10}\left(\frac{h}{m}\right)\left(3 \pi^{2}\right)^{2 / 3}$ and $c_{e}=e^{2} \frac{3}{4}\left(\frac{3}{\pi}\right)^{1 / 3}$. At TFW model (cf. [11],[12]), last term of TFDW theory at formula $(2.2)$ is in the form of gradient as $\frac{\hbar}{m} \int(\nabla \mathbf{n})^{1 / 2}(\mathbf{r})^{2} d \mathbf{r}$.

Notice that, for several nucleus, the external potential acting on each electron is $v_{\sigma}(t, \mathbf{r})=$ $v(\mathbf{r})+\delta v_{\sigma}(t, \mathbf{r})$, the exchange correction term $v(\mathbf{r})$ of columb potential at $E_{x c}$ is $v(\mathbf{r})=$ $\sum_{\sigma} \frac{C_{\sigma}}{\left|\mathbf{r}-R_{\sigma}\right|}, C_{\sigma}$ is electric charge, $R_{\sigma}$ is position, $\mathbf{r}$ is coordinate of electron at $\sigma$ th nucleus $R_{\sigma} . \delta v_{\sigma}$ is time-depended applied potential, $\delta v_{\sigma}=0$ at ground state $t=0$, and $\delta v_{\sigma}=$ $\vec{E}(t, \overrightarrow{\mathbf{r}}) \cdot \overrightarrow{\mathbf{r}}$ at $t \neq 0$.

Practically, consider the response to optical light, the electromagnetic field $\vec{E}(t, \overrightarrow{\mathbf{r}})$ (e.g. $=E(t) x \times \cos (k z-\omega t))$ and $\vec{B}(t, \overrightarrow{\mathbf{r}})$. The spatial variation of trapped field is negligible (at atomic unit, the distance from electron to nucleus, to photon are sufficient small, their forces are sufficient large), to be supposed spatial constant. At linear light response, dipole approximation to get $\delta v_{\sigma}(t, \mathbf{r})=\vec{E}(t, \overrightarrow{\mathbf{r}}) \cdot \overrightarrow{\mathbf{r}}$. It can be extended for response as Green's 
function, etc.

\section{Mathematical Setting}

For time-depended Schrödinger equation (2.1), to do mathematical setting in the framework of variational method at complex Hilbert spaces. For particles at matter or metallic surface, consider complex space for wave function in the sum form of $\mathbf{n}(t, \mathbf{r})$ on variable $t$ and $\mathbf{r}$, take complex Hilbert space in variational theory. In system $(2.1), \mathbf{g}(t)$ is external force of electron, as control input of particles. Control function $\mathbf{g}(t)$ at incident electric field $\vec{E}_{0}(t)$ is only depended on time $t$, and independent of spatial variable vector $\mathbf{r}$. $\mathbf{f}(t, \mathbf{r})$ is constrained force, a constrain on the particle to make it stay on the surface.

To open set $\Omega \subset \mathbf{R}^{2}$, and two usual spaces $L^{2}(\Omega)$ and $H_{0}^{1}(\Omega)$, define complex solution spaces $\mathbb{L}^{2}(\Omega)$ and $\mathbb{H}_{0}^{1}(\Omega)$. For complex-valued function $\mathbf{n}(t, \mathbf{r})=\sum_{i=1}^{N} \eta^{i}(t, \mathbf{r})$, denote two complex Hilbert spaces $\mathbb{H}=\mathbb{L}^{2}(\Omega)$ and $\mathbb{V}=\mathbb{H}_{0}^{1}(\Omega)$. Set $\mathbf{n}(t, \mathbf{r})=\sum_{i=1}^{N}\left(\eta^{i 1}+\mathbf{i} \eta^{i 2}\right) \in \mathbb{L}^{2}(\Omega)$ for $\eta^{i 1}, \eta^{i 2} \in L^{2}(\Omega)$, define norm of space $\mathbb{H}=\mathbb{L}^{2}(\Omega)$ as

$$
\|\mathbf{n}\|_{\mathbb{H}}=\sum_{i=1}^{N}\left(\left\|\eta^{i 1}\right\|_{L^{2}(\Omega)}^{2}+\left\|\eta^{i 2}\right\|_{L^{2}(\Omega)}^{2}\right)^{\frac{1}{2}} .
$$

Set $\mathbf{n}^{1}=\sum_{i=1}^{N} \eta^{i 1}$ and $\mathbf{n}^{2}=\sum_{i=1}^{n} \eta^{i 2}$, then $\mathbf{n}(t, \mathbf{r})=\mathbf{n}^{1}(t, \mathbf{r})+\mathbf{i} \mathbf{n}^{2}(t, \mathbf{r}) \in \mathbb{H}$. Similar to set $\overline{\mathbf{n}}^{1}=\sum_{i=1}^{N} \bar{\eta}^{i 1}$ and $\overline{\mathbf{n}}^{2}=\sum_{i=1}^{n} \bar{\eta}^{i 2}$, then $\overline{\mathbf{n}}(t, \mathbf{r})=\overline{\mathbf{n}}^{1}(t, \mathbf{r})+\mathbf{i} \overline{\mathbf{n}}^{2}(t, \mathbf{r}) \in \mathbb{H}$. Hence, the inner product of $\mathbb{H}$ can be defined by

$$
\begin{aligned}
(\mathbf{n}, \overline{\mathbf{n}})_{\mathbb{H}}= & \sum_{i=1}^{N}\left[\left(\eta^{i 1}, \bar{\eta}^{i 1}\right)_{L^{2}(\Omega)}+\left(\eta^{i 2}, \bar{\eta}^{i 2}\right)_{L^{2}(\Omega)}\right] \\
& +\mathbf{i} \sum_{i=1}^{N}\left[\left(\eta^{i 2}, \bar{\eta}^{i 1}\right)_{L^{2}(\Omega)}-\left(\eta^{i 1}, \bar{\eta}^{i 2}\right)_{L^{2}(\Omega)}\right] .
\end{aligned}
$$


For $\mathbf{n}(t, \mathbf{r})=\mathbf{n}^{1}(t, \mathbf{r})+\mathbf{i} \mathbf{n}^{2}(t, \mathbf{r}) \in \mathbb{V}$, define norm of $\mathbb{V}$ by

$$
\|\mathbf{n}\|_{\mathbb{V}}=\sum_{i=1}^{N}\left(\left\|\eta^{i 1}\right\|_{H_{0}^{1}(\Omega)}^{2}+\left\|\eta^{i 2}\right\|_{H_{0}^{1}(\Omega)}^{2}\right)^{\frac{1}{2}}
$$

If $\mathbf{n}, \overline{\mathbf{n}} \in \mathbb{V}$, then inner product of space $\mathbb{V}=\mathbb{H}_{0}^{1}(\Omega)$ is defined as

$$
(\mathbf{n}, \overline{\mathbf{n}})_{\mathbb{V}}=\sum_{i=1}^{N}\left(\eta^{i 1}, \bar{\eta}^{i 1}\right)_{H_{0}^{1}(\Omega)}+\sum_{i=1}^{N}\left(\eta^{i 2}, \bar{\eta}^{i 2}\right)_{H_{0}^{1}(\Omega)}
$$

The definition is equivalent to

$$
(\mathbf{n}, \overline{\mathbf{n}})_{\mathbb{V}}=\left(\nabla \eta^{i 1}, \nabla \bar{\eta}^{i 1}\right)_{\mathbb{L}^{2}(\Omega)}+\left(\nabla \eta^{i 2}, \nabla \bar{\eta}^{i 2}\right)_{\mathbb{L}^{2}(\Omega)}
$$

Remark 3. Notice that, without confusion, $H=L^{2}(\Omega), V=H_{0}^{1}(\Omega), \mathbb{H}=\mathbb{L}^{2}(\Omega), \mathbb{V}=\mathbb{H}_{0}^{1}(\Omega)$ indicate spatial variable at the orthogonal coordinate; $L^{2}(0, \infty)$ is for spatial variable $\mathbf{r}$ at the polar coordinate. There is no conflict at two coordinates.

Definition 4. The Hilbert space $W(0, T)$ is called solution space, defined by

$$
W(0, T)=\left\{\mathbf{n} \mid \mathbf{n} \in L^{2}(0, T ; \mathbb{V}), \mathbf{n}^{\prime} \in L^{2}\left(0, T ; \mathbb{V}^{\prime}\right)\right\}
$$

Then the norm of $W(0, T)$ can be defined as

$$
\|\mathbf{n}\|_{W(0, T)}=\sum_{i=1}^{N}\left(\left\|\eta^{i}\right\|_{L^{2}(0, T ; \mathbb{V})}^{2}+\left\|\eta^{i \prime}\right\|_{L^{2}\left(0, T ; \mathbb{V}^{\prime}\right)}^{2}\right)^{\frac{1}{2}}
$$

If $\mathbf{n}, \overline{\mathbf{n}} \in W(0, T)$, then the definition of its inner product is given by

$$
(\mathbf{n}, \overline{\mathbf{n}})_{W(0, T)}=\sum_{i=1}^{N}\left(\eta^{i}, \bar{\eta}^{i}\right)_{\mathbb{V}}+\left(\eta^{i \prime}, \bar{\eta}^{i \prime}\right)_{\mathbb{V}^{\prime}}
$$

Denotes continuous functions set $C(0, T ; \mathbb{H})$. Gelfand triple spaces $\mathbb{V} \hookrightarrow \mathbb{H} \hookrightarrow \mathbb{V}^{\prime}$, in which two embeddings are continuous, dense and compact. 


\section{Solution of Time-Depended Schrödinger Equation}

Definition 5. A function $\mathbf{n}$ is said as weak solution of the time-depended Schrödinger system (2.1) for $N$ particles, if $\mathbf{n} \in W(0, T)$ and satisfy

$$
\begin{aligned}
& \int_{0}^{T} \int_{\mathbf{i}} \mathbf{\partial} \frac{\partial \mathbf{n}(t, \mathbf{r})}{\partial t} \sigma d \mathbf{r} d t \\
= & -\frac{1}{2} \int_{0}^{T} \int \nabla \mathbf{n}(t, \mathbf{r}) \nabla \sigma d \mathbf{r} d t+\int_{0}^{T} \int[\mathbf{e}(t, \mathbf{r})+\mathbf{V}(t, \mathbf{r})-\mathbf{g}(t) \mathbf{r}+\mathbf{f}(t, \mathbf{r}) \mathbf{r}] \mathbf{n}(t, \mathbf{r}) \sigma d \mathbf{r} d t
\end{aligned}
$$

where $\forall \sigma \in \mathcal{D}^{\prime}(0, T)$ by the means of distribution space, $\sigma \in C^{1}(0, T ; \mathbb{V})$ and $\sigma(T)=0$. At time $t=0, \mathbf{n}(0, \mathbf{r})=\mathbf{n}_{0}(\mathbf{r})=\sum_{i=1}^{N} \eta^{i}(0, \mathbf{r})$.

Theorem 6. For $\mathbf{n}_{0}(\mathbf{r}) \in \mathbb{V}$ of $N$ particles, then there exists a unique weak solution $\mathbf{n}(t, \mathbf{r}) \in W(0, T)$ for time-depended Schrödinger system (2.1) in the sense of distribution in space $\mathcal{D}^{\prime}(0, T)$. The estimate for $\mathbf{n}(t, \mathbf{r})$ at the two complex Hilbert spaces is

$$
\|\mathbf{n}\|_{L^{2}(0, T ; \mathbb{H})}^{2}+\|\mathbf{n}\|_{L^{2}(0, T ; \mathbb{V})}^{2} \leq C\left(1+\left\|\mathbf{n}_{0}\right\|_{\mathbb{V}}^{2}+\|\mathbf{g}\|_{L^{2}(0, T)}^{2}+\|\mathbf{f}\|_{L^{2}\left(0, T ; L^{2}(0, \infty)\right)}^{2}\right)
$$

where $C$ is constant independent of $\mathbf{n}_{0}, \mathbf{g}$, and $\mathbf{f}$.

Proof. Faedo-Galerkin method could be using to construct an approximate solution for system (2.1). From Gelfand triple spaces, $\mathbb{V} \hookrightarrow \mathbb{H}$ is compact, then there exists an orthogonal basis of $\mathbb{H},\left\{w^{j}\right\}_{j=1}^{\infty}$ consisting of eigenfunctions of $A=-\Delta$, such that $A w^{j}=\lambda^{j} w^{j}$ for all $j, 0<\lambda^{1} \leq \lambda^{2} \leq, \cdots, \lambda^{j} \rightarrow \infty$ as $j \rightarrow \infty$. G $G^{i}$ is the orthogonal projection of $\mathbb{H}$ (or $\mathbb{V}$ ) onto the space spanned by $\left\{w^{1}, \cdots, w^{i^{\prime}}\right\}$. For $1 \leq i \leq N$, an approximate solution is defined for equation (2.1) by $\tilde{\eta}^{i j^{\prime}}(t)=\sum_{j=1}^{j^{\prime}} a^{i j}(t) w^{j}$, and $a^{i j}(t)$ is real-valued coefficient function. From $\mathbf{n}(t, \mathbf{r})=\sum_{i=1}^{N}\left|\eta^{i}(t, \mathbf{r})\right|^{2}$ to get expansion of $\tilde{e}(t, \mathbf{r})$ and $\tilde{V}(t, \mathbf{r})$ of $e(t, \mathbf{r})$ and $V(t, \mathbf{r})$ using $\left\{w^{j}\right\}$. For simplification, neglecting spatial variable $\mathbf{r}$ at the external and constrained forces, consider uniform optical fields $f(t)$ for $i$ th particle, taking $\sigma^{i}=w^{j}$ at weak form (4.1), then 
approximate solution $\tilde{\eta}^{i j^{\prime}}(t)$ satisfy ordinary differential equation as

$$
\left\{\begin{array}{l}
\int_{0}^{T} \int_{\mathbf{i}} \mathbf{i} \frac{\partial \tilde{\eta}^{i j^{\prime}}}{\partial t} w^{j} d \mathbf{r} d t=-\frac{1}{2} \int_{0}^{T} \int \nabla \tilde{\eta}^{i j^{\prime}} \nabla w^{j} d \mathbf{r} d t \\
+\int_{0}^{T} \int[\tilde{e}(t, \mathbf{r})+\tilde{V}(t, \mathbf{r})-g(t) \mathbf{r}+f(t) \mathbf{r}] \tilde{\eta}^{i j^{\prime}} w^{j} d \mathbf{r} d t \\
\tilde{\eta}^{i j^{\prime}}(0)=\tilde{\eta}_{0}^{i}, \quad 1 \leq i \leq N .
\end{array}\right.
$$

Substitute $\tilde{\eta}^{i j^{\prime}}$ as approximate form in (4.3), from standard theory of ODE, ensure a unique local solution $\left\{\tilde{\eta}^{i j^{\prime}}\right\}, i=1,2, \cdots, N$ for approximate system (4.3). For $\tilde{\eta}_{0}^{i j^{\prime}}$, there exists a $\bar{\eta}_{0}^{i} \in \mathbb{V}$ such that

$$
\tilde{\eta}_{0}^{i j^{\prime}} \rightarrow \bar{\eta}_{0}^{i} \text { strongly in } \quad \mathbb{H}_{0}^{1}(\Omega), \quad \text { as } j^{\prime} \rightarrow \infty
$$

Take analogous argument for $\left\{\tilde{\eta}^{i j^{\prime}}\right\}$, there exist a function $\bar{\eta}^{i} \in \mathbb{V}$, such that $\left\|\tilde{\eta}^{i j^{\prime}}-\bar{\eta}^{i}\right\|_{\mathbb{V}} \rightarrow 0$ as $j^{\prime} \rightarrow \infty$. That is, approximate solution $\tilde{\eta}^{i j^{\prime}}$ is bounded in $L^{\infty}(0, T ; \mathbb{V})$. Suppose that $\left\{\tilde{\eta}^{i j^{\prime}}\right\}$ and $\left\{\tilde{\eta}^{j j^{\prime}}\right\}$ are two solutions of equation (2.1) for initial guess $\eta_{0}^{i}$ and $\eta_{0}^{j}$, $\tilde{e}^{i j^{\prime}}=$ $\tilde{e}\left(t, \mathbf{r}, \eta^{i j^{\prime}}\right), \tilde{V}^{i j^{\prime}}=\tilde{V}\left(t, \mathbf{r}, \eta^{i j^{\prime}}\right)$ for solution $\eta^{i j^{\prime}}, \tilde{e}^{j j^{\prime}}=\tilde{e}\left(t, \mathbf{r}, \eta^{j j^{\prime}}\right), \tilde{V}^{j j^{\prime}}=\tilde{V}\left(t, \mathbf{r}, \eta^{j j^{\prime}}\right)$ for solution $\eta^{j j^{\prime}}$. Then to have

$$
\left\|\tilde{e}^{i j^{\prime}} \tilde{\eta}^{i j^{\prime}}-\tilde{e}^{j j^{\prime}} \tilde{\eta}^{j j^{\prime}}\right\|_{\mathbb{H}}^{2} \leq\left\|\tilde{e}^{i j^{\prime}}\right\|_{\mathbb{H}}^{2}+\left\|\tilde{\eta}^{i j^{\prime}}-\tilde{\eta}^{j j^{\prime}}\right\|_{\mathbb{H}}^{2}+\left\|\tilde{e}^{i j^{\prime}}-\tilde{e}^{j j^{\prime}}\right\|_{\mathbb{H}}^{2}+\left\|\tilde{\eta}^{j j^{\prime}}\right\|_{\mathbb{H}}^{2}
$$

There exist $C^{\prime}(t)$ such that

$$
\left\|\tilde{e}^{i j^{\prime}}-\tilde{e}^{j j^{\prime}}\right\|_{\mathbb{H}}^{2} \leq \int \frac{1}{\left|\mathbf{r}-\mathbf{r}^{\prime}\right|} \sum_{i=1}^{N}\left\|\tilde{\eta}^{i j^{\prime}}+\tilde{\eta}^{j j^{\prime}}\right\|_{\mathbb{H}}^{2}\left\|\eta^{i j^{\prime}}-\eta^{j j^{\prime}}\right\|_{\mathbb{H}}^{2} d \mathbf{r}^{\prime} \leq C^{\prime}(t)\left\|\tilde{\eta}^{i j^{\prime}}-\tilde{\eta}^{j j^{\prime}}\right\|_{\mathbb{H}}^{2}
$$

as $j^{\prime} \rightarrow \infty$. Further,

$$
\left\|\tilde{V}^{i j^{\prime}} \tilde{\eta}^{i j^{\prime}}-\tilde{V}^{j j^{\prime}} \tilde{\eta}^{j j^{\prime}}\right\|^{2} \leq\left\|\tilde{V}^{i j^{\prime}}\right\|_{\mathbb{H}}^{2}+\left\|\tilde{\eta}^{i j^{\prime}}-\tilde{\eta}^{j j^{\prime}}\right\|_{\mathbb{H}}^{2}+\left\|\tilde{V}^{i j^{\prime}}-\tilde{V}^{j j^{\prime}}\right\|_{\mathbb{H}}^{2}+\left\|\tilde{\eta}^{j j^{\prime}}\right\|_{\mathbb{H}}^{2}
$$


At TF model, there exist $C^{\prime \prime}(t)$ such that

$$
\left.\left\|\tilde{V}^{i j^{\prime}}-\tilde{V}^{j j^{\prime}}\right\|_{\mathbb{H}}^{2} \leq \frac{5}{3} c_{k} \int\left(\sum_{i=1}^{N}\left|\tilde{\eta}^{i j^{\prime}}(t, \mathbf{r})\right|^{2}\right)^{2 / 3}-\left(\sum_{j=1}^{N}\left|\tilde{\eta}^{j j^{\prime}}(t, \mathbf{r})\right|^{2}\right)^{2 / 3}\right) d \mathbf{r} \leq C^{\prime \prime}(t)\left\|\tilde{\eta}^{i j^{\prime}}-\tilde{\eta}^{j j^{\prime}}\right\|_{\mathbb{H}}^{2}
$$

Denote $\|\mathbf{r}\|$ as norm of vector $\mathbf{r}$, hence from weak form (4.1) to deduce

$$
\begin{aligned}
& \frac{1}{2} \frac{d}{d t}\left\|\tilde{\eta}^{i j^{\prime}}-\tilde{\eta}^{j j^{\prime}}\right\|_{\mathbb{H}}^{2}+g(t)\|\mathbf{r}\|\left\|\tilde{\eta}^{i j^{\prime}}-\tilde{\eta}^{j j^{\prime}}\right\|_{\mathbb{V}}^{2} \\
\leq & \left(-\frac{1}{2}+f(t)\|\mathbf{r}\|\right)\left\|\tilde{\eta}^{i j^{\prime}}-\tilde{\eta}^{j j^{\prime}}\right\|_{\mathbb{V}}^{2}+\left(1+C^{\prime}(t)+C^{\prime \prime}(t)\right)\left\|\tilde{\eta}^{i j^{\prime}}-\tilde{\eta}^{j j^{\prime}}\right\|_{\mathbb{H}}^{2} \\
& +\left(\left\|\tilde{e}^{i j^{\prime}}\right\|_{\mathbb{H}}^{2}+\left\|\tilde{V}^{i j^{\prime}}\right\|_{\mathbb{H}}^{2}+\left\|\tilde{\eta}^{j j^{\prime}}\right\|_{\mathbb{H}}^{2}\right)
\end{aligned}
$$

Set $I^{i j}(t)=\left(1+C^{\prime}(t)+C^{\prime \prime}(t)\right)\left\|\tilde{\eta}^{i j^{\prime}}-\tilde{\eta}^{j j^{\prime}}\right\|_{\mathbb{H}}^{2}$, since $\left(\frac{1}{2}+g(t)\|\mathbf{r}\|-f(t)\|\mathbf{r}\|\right)\left\|\tilde{\eta}^{i j^{\prime}}-\tilde{\eta}^{j j^{\prime}}\right\|_{\mathbb{V}}^{2} \geq 0$, by set $C(t)=\left\|\tilde{e}^{i j^{\prime}}\right\|_{\mathbb{H}}^{2}+\left\|\tilde{V}^{i j^{\prime}}\right\|_{\mathbb{H}}^{2}+\left\|\tilde{\eta}^{j j^{\prime}}\right\|_{\mathbb{H}}^{2}$ to convert that

$$
\frac{d}{d t} I^{i j}(t) \leq I^{i j}(t)+C(t)
$$

Set $I^{i j}(0)=\left(1+C^{\prime}(0)+C^{\prime \prime}(0)\right)\left\|\tilde{\eta}_{0}^{i j^{\prime}}-\tilde{\eta}_{0}^{j j^{\prime}}\right\|_{\mathbb{H}}^{2}$, Gronwall inequality yield that

$$
I^{i j}(t) \leq e^{t} I^{i j}(0)+\int_{0}^{t} C(t) e^{t-t^{\prime}} d t^{\prime}, \quad 0 \leq t^{\prime} \leq t
$$

Therefore, $\tilde{\eta}_{0}^{i j^{\prime}} \rightarrow \tilde{\eta}_{0}^{j j^{\prime}}$ implied $\tilde{\eta}^{i j^{\prime}} \rightarrow \tilde{\eta}^{j j^{\prime}}$ in $L^{2}(0, T ; \mathbb{H})$ as $j^{\prime} \rightarrow \infty$, and same argument to $L^{2}(0, T ; \mathbb{V})$ as $j^{\prime} \rightarrow \infty$ from formula (4.4). That is (4.2). It means that $\eta^{i}$ is bounded at complex Hilbert spaces $L^{2}(0, T ; \mathbb{H}) \cap L^{2}(0, T ; \mathbb{V})$. Taking $\tilde{\eta}^{j j^{\prime}}=\eta^{i}$ to find that $\tilde{\eta}^{i j^{\prime}} \rightarrow \eta^{i}$ in $C(0, T ; \mathbb{H})$. Thus, the inclusion $C(0, T ; \mathbb{H}) \subset W(0, T)$ to ensure Theorem 6 .

Remark 7. For sum function $\mathbf{n}(t, \mathbf{r})$ of time variable $t \in[0, T]$ and spatial variable $\mathbf{r} \in$ $(0, \infty)$, consider spatial depended external force $g(t, \mathbf{r})$ and constrained force $\mathbf{f}(t, \mathbf{r})$, complex parabolic differential equation (2.1) exist weak solution for ground state $\mathbf{n}_{0} \in \mathbb{V}$. 


\section{Control Theory for Particles at Surface}

Consider external control function $\mathbf{g}(t)$, and its quantum optimal control $\mathbf{g}^{*}(t)$. Suppose $\mathcal{G}=L^{2}(0, T)$ is the space of external control $\mathbf{g}(t, \mathbf{r})$, and $\mathcal{G}_{\text {ad }}$ is a admissible set of $\mathcal{G}$. Consider constrain control function $\mathbf{f}(t, \mathbf{r})$, and its quantum optimal control $\mathbf{f}^{*}(t, \mathbf{r})$. Suppose $\mathcal{F}=L^{2}\left(0, T ; L^{2}(0, \infty)\right)$ is the space of constrained control $\vec{F}(t, \overrightarrow{\mathbf{r}})$, and $\mathcal{F}_{a d}$ is a admissible set of $\mathcal{F}$. Set ground state of $N$ particles as $\mathbf{n}(0)=\mathbf{n}_{0}$. Consider cost function of $N$ particles for time-depended Schrödinger system (2.1) is in the form of

$$
J(\mathbf{g}, \mathbf{f})=\epsilon^{1}\left\|\mathbf{n}_{f}(\mathbf{g})-\mathbf{n}_{d}\right\|_{\mathbb{H}}^{2}+\epsilon^{2}\|\mathbf{g}\|_{\mathcal{G}}^{2}+\epsilon^{3}\|\mathbf{f}\|_{\mathcal{F}}^{2} .
$$

In criteria function (5.1), $\forall(\mathbf{g}, \mathbf{f}) \in \mathcal{G}_{a d} \times \mathcal{F}_{a d}, \mathbf{n}_{d}$ is target state, $\mathbf{n}_{f}(\mathbf{g})$ is observed final state of particles at final time $t_{f} \cdot \epsilon^{i}, i=1,2,3$ are weighted coefficients for balancing the values of inherent cost and running cost.

Quantum optimal control for system (2.1) is to solve two fundamental problems:

i). find quantum optimal control $\left(\mathbf{g}^{*}, \mathbf{f}^{*}\right)$ for the system (2.1) such that.

$$
J\left(\mathbf{g}^{*}, \mathbf{f}^{*}\right)=\inf _{(\mathbf{g}, \mathbf{f}) \in \mathcal{G}_{a d} \times \mathcal{F}_{a d}} J(\mathbf{g}, \mathbf{f}) .
$$

ii). find Euler-Lagrange system to characterize $\left(\mathbf{g}^{*}, \mathbf{f}^{*}\right)$.

For $N$ particles, $\left(\mathbf{g}^{*}(t), \mathbf{f}^{*}(t, \mathbf{r})\right)$ is called quantum optimal control for time-depended Schrödinger system (2.1) subject to cost function (5.1).

To control variable $(\mathbf{g}, \mathbf{f})$, by the virtual of Theorem 5 , there is a unique weak solution $\mathbf{n}(t, \mathbf{r}) \in W(0, T)$ of the system (2.1) subject to cost (5.1). Therefore, there exist a continuous mapping $(\mathbf{g}, \mathbf{f}) \rightarrow \mathbf{n}(t, \mathbf{r})$ from control space $\mathcal{G} \times \mathcal{F}$ to solution space $W(0, T)$.

Theorem 8. For $\mathbf{n}_{0} \in \mathbb{V}$ of $N$ particles, if $\mathcal{G}_{a d} \times \mathcal{F}_{a d}$ is closed convex (bounded) admissible subset of $\mathcal{G} \times \mathcal{F}$, then there exists at least one quantum optimal control $\left(\mathbf{g}^{*}, \mathbf{f}^{*}\right)$ of timedepended Schrödinger system (2.1) subject to cost function (5.1). 
Proof. Set $J=\inf _{(\mathbf{g}, \mathbf{f}) \in \mathcal{G}_{a d} \times \mathcal{F}_{a d}} J(\mathbf{g}, \mathbf{f})$. Since $\mathcal{G}_{a d} \times \mathcal{F}_{a d}$ is non-empty, closed and convex (bounded) subset of $\mathcal{G} \times \mathcal{F}$, there is a sequence $\left\{\left(\mathbf{g}^{k}, \mathbf{f}^{k}\right)\right\}$ in $\mathcal{G}_{a d} \times \mathcal{F}_{a d}$ such that $\inf _{(\mathbf{g}, \mathbf{f}) \in \mathcal{G}_{a d} \times \mathcal{F}_{a d}} J(\mathbf{g}, \mathbf{f})=$ $\lim _{k \rightarrow \infty} J\left(\mathbf{g}^{k}, \mathbf{f}^{k}\right)=J$. Since $\{J(\mathbf{g}, \mathbf{f})\}$ is bounded in $\mathbf{R}^{+}$, there exist a subsequences $\left\{\left(\mathbf{g}^{k^{\prime}}, \mathbf{f}^{k^{\prime}}\right)\right\}$ of $\left\{\left(\mathbf{g}^{k}, \mathbf{f}^{k^{\prime}}\right)\right\}$ can be extracted, and exist a $\left(\mathbf{g}^{*}, \mathbf{f}^{*}\right) \in \mathcal{G}_{a d} \times \mathcal{F}_{a d}$, such that

$$
\left(\mathbf{g}^{k^{\prime}}, \mathbf{f}^{k^{\prime}}\right) \rightarrow\left(\mathbf{g}^{*}, \mathbf{f}^{*}\right) \text { weakly in } \mathcal{G} \times \mathcal{F} \text { as } k^{\prime} \rightarrow \infty
$$

From Theorem 5 to get estimate $\|\mathbf{n}\|_{\mathbb{H}}^{2}+\|\mathbf{n}\|_{\mathbb{V}}^{2}$ bounded for $\mathbf{n}$ of $N$ particles. For control $\left(\mathbf{g}^{k^{\prime}}, \mathbf{f}^{k^{\prime}}\right)$, from boundedness of $\mathcal{G}_{a d} \times \mathcal{F}_{a d}$ that

$$
\mathbf{n}\left(\mathbf{g}^{k^{\prime}}, \mathbf{f}^{k^{\prime}}\right) \text { is bounded in } L^{2}(0, T ; \mathbb{H}) \cap L^{2}(0, T ; \mathbb{V})
$$

Setting $\mathbf{n}^{*}=\mathbf{n}\left(\mathbf{g}^{*}, \mathbf{f}^{*}\right)$, there exist a subsequence $\left\{\mathbf{n}\left(\mathbf{g}^{k^{\prime \prime}}, \mathbf{f}^{k^{\prime \prime}}\right)\right\}$ of $\left\{\mathbf{n}\left(\mathbf{g}^{k^{\prime}}, \mathbf{f}^{k^{\prime}}\right)\right\}$, and $\mathbf{n}^{*} \in$ $W(0, T)$ such that

$$
\mathbf{n}\left(\mathbf{g}^{k^{\prime \prime}}, \mathbf{f}^{k^{\prime \prime}}\right) \rightarrow \mathbf{n}^{*} \text { weakly in } L^{2}(0, T ; \mathbb{H}) \cap L^{2}(0, T ; \mathbb{V})
$$

as $k^{\prime \prime} \rightarrow \infty$. Since embedding $\mathbb{V} \hookrightarrow \mathbb{H}$ is compact, from Aubin-Lions-Temam theorem, there is $\overline{\mathbf{n}} \in \mathbb{H}$ s.t.

$$
\mathbf{n}\left(\mathbf{g}^{k^{\prime \prime}}, \mathbf{f}^{k^{\prime \prime}}\right) \rightarrow \overline{\mathbf{n}} \text { strongly in } L^{2}(0, T ; \mathbb{H}), \text { as } k^{\prime \prime} \rightarrow \infty,
$$

and get the convergences for $\mathbf{n}\left(\mathbf{g}^{k^{\prime \prime}}, \mathbf{f}^{k^{\prime \prime}}\right)$ as

$$
\begin{aligned}
& \frac{\partial \mathbf{n}\left(\mathbf{g}^{k^{\prime \prime}}, \mathbf{f}^{k^{\prime \prime}}\right)}{\partial t} \rightarrow \frac{\partial \overline{\mathbf{n}}}{\partial t} \quad \text { weakly in } L^{2}\left(0, T ; \mathbb{V}^{\prime}\right) \\
& \nabla \mathbf{n}\left(\mathbf{g}^{k^{\prime \prime}}, \mathbf{f}^{k^{\prime \prime}}\right) \rightarrow \nabla \overline{\mathbf{n}} \quad \text { weakly in } L^{2}(0, T ; \mathbb{H}),
\end{aligned}
$$


as $k^{\prime \prime} \rightarrow \infty$. Set $\mathbf{n}^{k^{\prime \prime}}=\mathbf{n}\left(\mathbf{g}^{k^{\prime \prime}}, \mathbf{f}^{k^{\prime \prime}}\right), \mathbf{n}_{0}^{k^{\prime \prime}}=\mathbf{n}_{0}$, therefore, for $N$ particles, taking test function $w^{j}=\sigma$ in weak form of approximation solution, by definition of weak form (4.1) to find

$$
\left\{\begin{array}{l}
\int_{0}^{T} \int_{0}^{T} \mathbf{i} \frac{\mathbf{n}^{k^{\prime \prime}}}{\partial t} \sigma d \mathbf{r} d t=-\frac{1}{2} \int_{0}^{T} \int \nabla \mathbf{n}^{k^{\prime \prime}} \nabla \sigma d \mathbf{r} d t \\
+\int_{0}^{T} \int[\mathbf{e}(t, \mathbf{r})+\mathbf{V}(t, \mathbf{r})-\mathbf{g}(t) \mathbf{r}+\mathbf{f}(t, \mathbf{r}) \mathbf{r}] \mathbf{n}^{k^{\prime \prime}} \sigma d \mathbf{r} d t \\
\mathbf{n}^{k^{\prime \prime}}(0)=\mathbf{n}_{0}^{k^{\prime \prime}}
\end{array}\right.
$$

If using (5.2), (5.3), (5.4), and taking $k^{\prime \prime} \rightarrow \infty$ to yield that

$$
\left\{\begin{array}{l}
\int_{0}^{T} \int_{0} \mathbf{i} \frac{\partial \overline{\mathbf{n}}}{\partial t} \sigma d \mathbf{r} d t=-\frac{1}{2} \int_{0}^{T} \int \nabla \overline{\mathbf{n}} \nabla \sigma d \mathbf{r} d t \\
+\int_{0}^{T} \int[\mathbf{e}(t, \mathbf{r})+\mathbf{V}(t, \mathbf{r})-\mathbf{g}(t) \mathbf{r}+\mathbf{f}(t, \mathbf{r}) \mathbf{r}] \overline{\mathbf{n}} \sigma d \mathbf{r} d t \\
\overline{\mathbf{n}}(0)=\mathbf{n}_{0}, \quad \forall \sigma \in C^{1}(0, T ; \mathbb{V})
\end{array}\right.
$$

It inferred that $\overline{\mathbf{n}}$ is a solution of (2.1) in the sense of distribution $\mathcal{D}^{\prime}(0, T)$ on $(0, T)$. From uniqueness of weak solution for system (2.1) to attain $\overline{\mathbf{n}}=\mathbf{n}\left(\mathbf{g}^{*}, \mathbf{f}^{*}\right)$. That is, for $\mathbf{n}^{*}$ to get two convergences as

$$
\begin{aligned}
& \mathbf{n}\left(\mathbf{g}^{k^{\prime \prime}}, \mathbf{f}^{k^{\prime \prime}}\right) \rightarrow \mathbf{n}\left(\mathbf{g}^{*}, \mathbf{f}^{*}\right) \quad \text { strongly in } L^{2}(0, T ; \mathbb{H}) \\
& \mathbf{n}_{f}\left(\mathbf{g}^{k^{\prime \prime}}, \mathbf{f}^{k^{\prime \prime}}\right) \rightarrow \mathbf{n}_{f}\left(\mathbf{g}^{*}, \mathbf{f}^{*}\right) \quad \text { strongly in } \mathbb{H},
\end{aligned}
$$

as $k^{\prime \prime} \rightarrow \infty$. Since the norm $\|\cdot\|_{\mathbb{L}^{2}(\Omega)}$ are lower semi-continuous in weak topology of $\mathbb{L}^{2}(\Omega)$, for $N$ particles

$$
\liminf _{k^{\prime \prime} \rightarrow \infty}\left\|\mathbf{n}_{f}\left(\mathbf{g}^{k^{\prime \prime}}, \mathbf{f}^{k^{\prime \prime}}\right)-\mathbf{n}_{d}\right\|_{\mathbb{H}}^{2} \geq\left\|\mathbf{n}_{f}\left(\mathbf{g}^{*}, \mathbf{f}^{*}\right)-\mathbf{n}_{d}\right\|_{\mathbb{H}}^{2}
$$

Vice versa, from weak convergence () that

$$
\liminf _{k^{\prime \prime} \rightarrow \infty}\left(\mathbf{g}^{k^{\prime \prime}}, \mathbf{g}^{k^{\prime \prime}}\right)_{\mathcal{G}} \geq\left(\mathbf{g}^{*}, \mathbf{g}^{*}\right)_{\mathcal{G}}, \liminf _{k^{\prime \prime} \rightarrow \infty}\left(\mathbf{f}^{k^{\prime \prime}}, \mathbf{f}^{k^{\prime \prime}}\right)_{\mathcal{F}} \geq\left(\mathbf{f}^{*}, \mathbf{f}^{*}\right)_{\mathcal{F}}
$$


For cost function (5.1), $J=\liminf _{k^{\prime \prime} \rightarrow \infty} J\left(\mathbf{g}^{k^{\prime \prime}}, \mathbf{f}^{k^{\prime \prime}}\right) \geq J\left(\mathbf{g}^{*}, \mathbf{f}^{*}\right)$, and $J\left(\mathbf{g}^{*}, \mathbf{f}^{*}\right)=\inf _{(\mathbf{g}, \mathbf{f}) \in \mathcal{G}_{a d} \times \mathcal{F}_{a d}} J(\mathbf{g}, \mathbf{f})$. That is, $\left(\mathbf{g}^{*}, \mathbf{f}^{*}\right)$ is quantum optimal control of time-depended Schrödinger system (2.1) subject to criteria function (5.1). It is Theorem 8 .

Theorem 9. For $\mathbf{n}_{0} \in \mathbb{V}$ of control problem for system (2.1) to cost function (5.1), if $\mathcal{G}_{a d} \times \mathcal{F}_{a d}$ is closed convex (bounded) admissible subset of $\mathcal{G} \times \mathcal{F}$, then quantum optimal control $\left(\mathbf{g}^{*}, \mathbf{f}^{*}\right)$ is characterized by Euler-Lagrange (optimality) system as

$$
\begin{aligned}
& \left\{\begin{array}{l}
\mathbf{i} \frac{\partial}{\partial t} \mathbf{n}(t, \mathbf{r})=-\frac{1}{2} \nabla^{2} \mathbf{n}(t, \mathbf{r})+[\mathbf{e}(t, \mathbf{r})+\mathbf{V}(t, \mathbf{r}) \\
\left.-\mathbf{g}^{*}(t) \mathbf{r}+\mathbf{f}^{*}(t, \mathbf{r}) \mathbf{r}\right] \mathbf{n}(t, \mathbf{r}) \text { in } \mathrm{Q}, \\
\mathbf{n}\left(0, \mathbf{g}^{*}\right)=\mathbf{n}_{0} \text { on } \Omega .
\end{array}\right. \\
& \left\{\begin{array}{c}
\mathbf{i} \frac{\partial}{\partial t} \tilde{\mathbf{n}}(t, \mathbf{r})=-\frac{1}{2} \nabla^{2} \tilde{\mathbf{n}}(t, \mathbf{r})+[\mathbf{e}(t, \mathbf{r})+\mathbf{V}(t, \mathbf{r})] \tilde{\mathbf{n}}(t, \mathbf{r}) \text { in } \mathrm{Q}, \\
\mathbf{i} \tilde{\mathbf{n}}\left(T, \mathbf{g}^{*}\right)=\mathbf{n}_{f}\left(\mathbf{g}^{*}, \mathbf{f}^{*}\right)-\mathbf{n}_{d} \text { on } \Omega .
\end{array}\right. \\
& \int_{0}^{T} \int \sigma^{*} \tilde{\mathbf{n}}\left(\mathbf{g}^{*}, \mathbf{f}^{*}\right)\left(\mathbf{g}+\mathbf{f}-\mathbf{g}^{*}-\mathbf{f}^{*}\right) d \mathbf{r} d t \\
& +\left(\mathbf{g}^{*}, \mathbf{g}-\mathbf{g}^{*}\right)_{\mathcal{G}}+\left(\mathbf{f}^{*}, \mathbf{f}-\mathbf{f}^{*}\right)_{\mathcal{F}} \geq 0, \forall(\mathbf{g}, \mathbf{f}) \in \mathcal{G}_{a d} \times \mathcal{F}_{a d}
\end{aligned}
$$

In here, $\tilde{\mathbf{n}}(t, \mathbf{r}) \in W(0, T)$ is weak solution of adjoint system (5.6) to $\mathbf{n}(t, \mathbf{r})$ of system (5.5). (5.7) is necessary optimality condition for quantum optimal control $\mathbf{g}^{*}$ and $\mathbf{f}^{*}$ of $N$ particles. $\sigma^{*}$ is conjugate operator of $\sigma$ in $\sigma \tilde{\mathbf{n}}=\left(\mathbf{g}^{*}+\mathbf{f}^{*}\right) \tilde{\mathbf{n}}$.

\section{Conclusions and discussions}

In this work, theoretical quantum control for trapped particles at matter surface has been considered using density function theory (DFT). For time-depended Schrödinger equation of $N$ particles, we are applying quantum control theory to quantum system of electron motion surrounding particles.

For particles appeared at 2D plane surface, the existence of solution of trapped quantum system is proved by variational method in complex Hilbert spaces. For external force and 
constrained force acted at the $N$ particles at matter surface, the existence of optimal control and its characterized optimality system had been investigated.

Computational and experimental control would be the future work of controlling particles at surface having optical equipments at laboratory at the viewpoint of physical and chemical fields.

\section{Acknowledgement}

The author sincere thanks to 250th ACS National Meeting \& Exposition 2015 for poster abstract 592 at PHYS Poster session.

\section{References}

(1) Q. F. Wang, Quantum control at matter surface, ACS National Meeting \& Exposition 2009, Poster.

(2) Q. F. Wang, Quantum control for particles at matter surface, ACS National Meeting \& Exposition 2014, Poster abstract.

(3) Q. F. Wang, Quantum numerical control for particles at matter surface, 249th ACS National Meeting \& Exposition 2015, Poster abstract.

(4) H. G Mack, Combined quantum mechanics and classical electrodynamics mulit-scale approach for the calculation of SERS spectra (A brief survey), 2013.

(5) J. D. Morgan III, Thomas-Fermi and other density function theories, Atomic, Molecular, \& Optical Physics Handbooks, 1996.

(6) N. M. Harrison, An Introduction to Density Function Theory, 2002.

(7) L. H. Thomas, The calculation of atomic fields, Proc. Camb. Phil. Soc. 23, 542, 1927. 
(8) E. Fermi, Un Metodo Statistico per la Determinazione di alcune Prioprietà dell'Atomo, Rend. Accad. Naz. Lincei 6,602, 1927.

(9) P. A. M. Dirac, Note on exchange phenomena in the Thomas atom, Proc. Camb. Phil. Soc. 26, 376, 1930 .

(10) C. F. Weizsäcker, Zur Theorie der Kernmassen, Zeitschrift für Physik 96 (7-8) 431, 1935.

(11) P. Hohenberg, W. Kohn, Inhomogeneous electron gad, Physical Review 136, B864, 1964.

(12) W. Kohn, I, J. Sham, Self-consistent equations including exchange and correlation effects, Physical Review 140, A1133, 1965. 